\title{
More on the hypercharge portal into the dark sector
}

\section{Florian Domingo, ${ }^{a}$ Oleg Lebedev, ${ }^{a}$ Yann Mambrini, ${ }^{b}$ Jérémie Quevillon ${ }^{b}$ and Andreas Ringwald ${ }^{a}$}

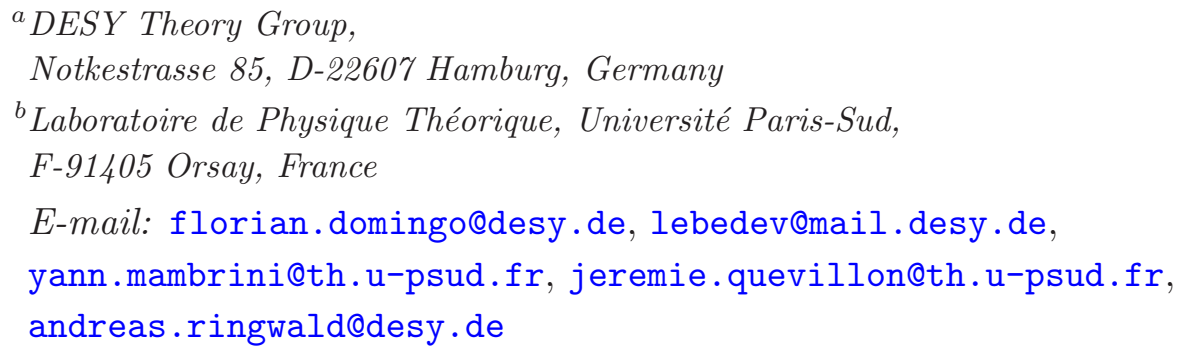

ABSTRACT: If the hidden sector contains more than one U(1) groups, additional dim-4 couplings (beyond the kinetic mixing) between the massive $\mathrm{U}(1)$ fields and the hypercharge generally appear. These are of the form similar to the Chern-Simons interactions. We study the phenomenology of such couplings including constraints from laboratory experiments and implications for dark matter. The hidden vector fields can play the role of dark matter whose characteristic signature would be monochromatic gamma ray emission from the galactic center. We show that this possibility is consistent with the LHC and other laboratory constraints, as well as astrophysical bounds.

Keywords: Beyond Standard Model, Cosmology of Theories beyond the SM, Gauge Symmetry

ARXIV EPRINT: 1305.6815 


\section{Contents}

1 Introduction 1

2 Hypercharge couplings to the "hidden" sector 2

3 Phenomenological constraints $\quad 4$

3.1 Unitarity 4

3.2 Invisible $\boldsymbol{\Upsilon}$ decay 4

3.3 Invisible $Z$ decay 5

$3.4 B \rightarrow K+$ inv and $K \rightarrow \pi+$ inv $\quad 6$

3.5 Bounds on decaying vector bosons $D \rightarrow C+\gamma \quad 7$

4 Vector Dark Matter and the Chern-Simons coupling 9

$\begin{array}{lll}4.1 & \text { WMAP/PLANCK constraints } & 10\end{array}$

$\begin{array}{lll}4.2 & \text { Indirect DM detection constraints } & 12\end{array}$

4.3 On the tentative $135 \mathrm{GeV}$ gamma-ray line 13

$\begin{array}{lll}4.4 & \text { Direct detection constraints } & 14\end{array}$

$\begin{array}{ll}\text { 4.5 LHC monojet constraints } & 14\end{array}$

$\begin{array}{lll}4.6 & \text { LHC monophoton constraints } & 16\end{array}$

$\begin{array}{ll}4.7 \text { Summary of constraints } & 17\end{array}$

$\begin{array}{llr}5 & \text { Conclusion } & 18\end{array}$

\section{Introduction}

The existence of new physics structures beyond those of the Standard Model (SM) is motivated, among other things, by the puzzles of dark matter (DM) and inflation. The minimal way to address these problems is to add a "hidden" sector containing the required SM-singlet fields. The existence of the hidden sector can also be motivated from the topdown viewpoint, in particular, by realistic string constructions [1, 2]. Such a sector can couple to the SM fields through products of gauge-singlet operators, including those of dimension 2 and 3. In this work, we study in detail the corresponding couplings to the hypercharge field.

Let us define the "hidden sector" as a set of fields which carry no SM gauge quantum numbers. Then a "portal" [3] would be an operator that couples the SM fields to such SM singlets. Let us consider the minimal case: suppose that the relevant low energy degrees of freedom in the hidden sector are those of a Weyl fermion $\chi$, or a massive vector $V_{\mu}$, or 
a real scalar $S$ (one field at a time). Then the lowest, up to dim-4, dimension operators which couple the SM to the hidden sector are given by

$$
\begin{aligned}
& O_{1}=\Psi_{L} H \chi+\text { h.c. } \\
& O_{2}=F_{\mu \nu}^{Y} F^{V \mu \nu}, \\
& O_{3}=\bar{\Psi}_{i} \gamma_{\mu}\left(1+\alpha_{i j} \gamma_{5}\right) \Psi_{j} V^{\mu}+\text { h.c. } \\
& O_{4}=H^{\dagger} H V_{\mu} V^{\mu}+\beta H^{\dagger} i D_{\mu} H V^{\mu}+\text { h.c. }, \\
& O_{5}=H^{\dagger} H S^{2}+\mu_{S} H^{\dagger} H S .
\end{aligned}
$$

Here $\Psi_{L}$ is the lepton doublet; $F_{\mu \nu}^{Y}$ and $F_{\mu \nu}^{V}$ are the field strength tensors for hypercharge and $V_{\mu}$, respectively; $\Psi_{i}$ is an SM fermion with generation index $i ; D_{\mu}$ is the covariant derivative with respect to the SM gauge symmetries, and $\alpha_{i j}, \beta, \mu_{S}$ are constants. Note that a particular version of operator $O_{3}$ is induced by $O_{2}$ after diagonalization of the vector kinetic terms.

An attractive feature of such an extension of the Standard Model is that it can offer viable dark matter candidates as well as provide a link to the inflaton sector. In particular, a sufficiently light "right-handed neutrino" $\chi$ is long-lived and can constitute warm dark matter [4]. Also, a massive vector $V_{\mu}$ (or a scalar $S$ [5]) can inherit a $Z_{2}$ symmetry from hidden sector gauge interactions, which would eliminate terms linear in $V_{\mu}$ and make it a stable cold dark matter candidate [6]. Finally, the Higgs coupling $H^{\dagger} H S^{2}$ to the inflaton $S$ would be instrumental in reconciling metastability of the electroweak vacuum with inflation [7].

In this work, we explore a more general dim-4 hypercharge coupling to the hidden sector, when the latter contains multiple U(1)'s. In this case, a Chern-Simons-type coupling becomes possible [8-13]. If such a coupling is the only SM portal into the hidden sector, the lightest $\mathrm{U}(1)$ vector field can play the role of dark matter. The trademark signature of this scenario is the presence of monochromatic gamma-ray lines in the photon spectrum of the galactic center. We analyze general experimental constraints on the Chern-Simons-type coupling as well as the constraints applicable when the vector field constitutes dark matter.

\section{Hypercharge couplings to the "hidden" sector}

Suppose the "hidden" sector contains two massive U(1) gauge fields $C_{\mu}$ and $D_{\mu}$. Before electroweak symmetry breaking, the most general dim- 4 interactions of these fields with the hypercharge boson $B_{\mu}$ are described by the Lagrangian

$$
\begin{aligned}
\mathcal{L}= & -\frac{1}{4} B_{\mu \nu} B^{\mu \nu}-\frac{1}{4} C_{\mu \nu} C^{\mu \nu}-\frac{1}{4} D_{\mu \nu} D^{\mu \nu}-\frac{\delta_{1}}{2} B_{\mu \nu} C^{\mu \nu}-\frac{\delta_{2}}{2} B_{\mu \nu} D^{\mu \nu}-\frac{\delta_{3}}{2} C_{\mu \nu} D^{\mu \nu} \\
& +\frac{M_{C}^{2}}{2} C_{\mu} C^{\mu}+\frac{M_{D}^{2}}{2} D_{\mu} D^{\mu}+\delta M^{2} C_{\mu} D^{\mu}+\kappa \epsilon_{\mu \nu \rho \sigma} B^{\mu \nu} C^{\rho} D^{\sigma}
\end{aligned}
$$

Here we have assumed CP symmetry such that terms of the type $B^{\mu \nu} C_{\mu} D_{\nu}$ are not allowed (see [14] for a study of the latter). The kinetic and mass mixing can be eliminated by field 
redefinition [15], which to first order in the mixing parameters $\delta_{i}$ and $\delta M^{2}$ reads

$$
\begin{aligned}
& B_{\mu} \rightarrow B_{\mu}+\delta_{1} C_{\mu}+\delta_{2} D_{\mu}, \\
& C_{\mu} \rightarrow C_{\mu}+\frac{\delta_{3} M_{D}^{2}-\delta M^{2}}{M_{D}^{2}-M_{C}^{2}} D_{\mu}, \\
& D_{\mu} \rightarrow D_{\mu}-\frac{\delta_{3} M_{C}^{2}-\delta M^{2}}{M_{D}^{2}-M_{C}^{2}} C_{\mu} .
\end{aligned}
$$

In terms of the new fields, the Lagrangian is

$$
\mathcal{L}=-\frac{1}{4} B_{\mu \nu} B^{\mu \nu}-\frac{1}{4} C_{\mu \nu} C^{\mu \nu}-\frac{1}{4} D_{\mu \nu} D^{\mu \nu}+\frac{M_{C}^{2}}{2} C_{\mu} C^{\mu}+\frac{M_{D}^{2}}{2} D_{\mu} D^{\mu}+\kappa \epsilon_{\mu \nu \rho \sigma} B^{\mu \nu} C^{\rho} D^{\sigma},
$$

which will be the starting point for our phenomenological analysis. We note that, due to the kinetic mixing $\delta_{1,2}, C_{\mu}$ and $D_{\mu}$ have small couplings to the Standard Model matter. Since we are mainly interested in the effect of the Chern-Simons-type term $\epsilon_{\mu \nu \rho \sigma} B^{\mu \nu} C^{\rho} D^{\sigma}$, we will set $\delta_{1,2}$ to be very small or zero in most of our analysis.

The term $\epsilon_{\mu \nu \rho \sigma} B^{\mu \nu} C^{\rho} D^{\sigma}$ has dimension 4 . However, it vanishes in the limit of zero vector boson masses by gauge invariance, both for the Higgs and Stückelberg mechanisms. This means that it comes effectively from a higher dimensional operator with $\kappa$ proportional to $M_{C} M_{D} / \Lambda^{2}$, where $\Lambda$ is the cutoff scale or the mass scale of heavy particles we have integrated out. On one hand, this operator does not decouple as $\Lambda \rightarrow \infty$ since both $M_{C, D}$ and $\Lambda$ are given by the "hidden" Higgs VEV times the appropriate couplings; on the other hand, $\epsilon_{\mu \nu \rho \sigma} B^{\mu \nu} C^{\rho} D^{\sigma}$ is phenomenologically relevant only if $M_{C, D}$ are not far above the weak scale. Thus, this term represents a meaningful approximation in a particular energy window, which we will quantify later. (A similar situation occurs in the vector Higgs portal models, where the interaction $H^{\dagger} H V_{\mu} V^{\mu}$ has naive dimension 4, but originates from a dim-6 operator [6].) From the phenomenological perspective, it is important that $\epsilon_{\mu \nu \rho \sigma} B^{\mu \nu} C^{\rho} D^{\sigma}$ is the leading operator at low energies, e.g. relevant to non-relativistic annihilation of dark matter composed of $C_{\mu}$ or $D_{\mu}$, and thus we will restrict our attention to this coupling only.

A coupling of this sort appears in various models upon integrating out heavy fields charged under both U(1)'s and hypercharge. Explicit anomaly-free examples can be found in $[11,12]$ and $[10]$. In these cases, the Chern-Simons term arises upon integrating out heavy, vector-like with respect to the SM, fermions. Both the vectors and the fermions get their masses from the Higgs mechanism, while the latter can be made heavy by choosing large Yukawa couplings compared to the gauge couplings. In this limit, eq. (2.3) gives the corresponding low energy action. ${ }^{1}$

Finally, we note that increasing the number of hidden U(1)'s does not bring in hypercharge-portal interactions with a new structure, so our considerations apply quite generally.

\footnotetext{
${ }^{1}$ We note that certain "genuine" gauge invariant dim-6 operators such as $\frac{1}{\Lambda^{2}} \epsilon^{\mu \nu \rho \sigma} B_{\mu \nu} C_{\rho}^{\tau} D_{\tau \sigma}$ reduce to the Chern-Simons term on-shell in the non-relativistic limit $\left(C_{\mu \nu} \rightarrow C_{0 i}=i M_{C} C_{i} ; C_{0}=0\right.$ and similarly for $\left.D_{\mu \nu}\right)$. Such operators should generally be taken into account when deriving the low energy action in explicit microscopic models.
} 


\section{Phenomenological constraints}

In this section we derive constraints on the coupling constant $\kappa$ from various laboratory experiments as well as unitarity considerations. The relevant interaction to leading order is given by

$$
\Delta \mathcal{L}=\kappa \cos \theta_{W} \epsilon_{\mu \nu \rho \sigma} F^{\mu \nu} C^{\rho} D^{\sigma}-\kappa \sin \theta_{W} \epsilon_{\mu \nu \rho \sigma} Z^{\mu \nu} C^{\rho} D^{\sigma},
$$

where $F^{\mu \nu}$ and $Z^{\mu \nu}$ are the photon and Z-boson field strengths, respectively.

In what follows, we set the kinetic mixing to be negligibly small such that the lighter of the $C$ and $D$ states is not detected and thus appears as missing energy and momentum. There are then two possibilities: the heavier state decays into the lighter state plus $\gamma$ either outside or inside the detector. Consider first the case where the mass splitting and $\kappa$ are relatively small such that both $C$ and $D$ are "invisible".

\subsection{Unitarity}

The coupling $\epsilon_{\mu \nu \rho \sigma} B^{\mu \nu} C^{\rho} D^{\sigma}$ involves longitudinal components of the massive vectors. Therefore, some scattering amplitudes will grow indefinitely with energy, which imposes a cutoff on our effective theory. For a fixed cutoff, this translates into a bound on $\kappa$.

Consider the scattering process

$$
C_{\mu} C_{\nu} \rightarrow D_{\rho} D_{\sigma}
$$

at high energies, $E \gg M_{C, D}$. The vertex can contain longitudinal components of at most one vector since $\epsilon_{\mu \nu \rho \sigma}\left(p_{1}+p_{2}\right)^{\mu} p_{1}^{\nu} p_{2}^{\rho}=0$. Then one finds that the amplitude grows quadratically with energy,

$$
\mathcal{A} \sim \kappa^{2} \frac{E^{2}}{M_{C, D}^{2}}
$$

with the subscripts $C$ and $D$ applying to the processes involving longitudinal components of $C_{\mu}$ and $D_{\mu}$, respectively. On the other hand, the amplitude cannot exceed roughly $8 \pi$. Neglecting order one factors, the resulting constraint is

$$
\frac{\kappa}{M}<\frac{\sqrt{8 \pi}}{\Lambda}
$$

where $M=\min \left\{M_{C}, M_{D}\right\}$ and $\Lambda$ is the cutoff scale. As explained in the previous section, $\Lambda$ is associated with the mass scale of new states charged under $\mathrm{U}(1)_{\mathrm{Y}}$. Since constraints on such states are rather stringent, it is reasonable to take $\Lambda \sim 1 \mathrm{TeV}$. This implies that light vector bosons can couple only very weakly, e.g. $\kappa<10^{-5}$ for $M \sim 1 \mathrm{MeV}$.

It is important to note that the unitarity bound applies irrespective of whether $C$ and $D$ are stable or not. Thus it applies to the case $M_{D} \gg M_{C}$ or vice versa and also in the presence of the kinetic mixing.

\subsection{Invisible $\Upsilon$ decay}

Suppose that $D$ is the heavier state and the decay $D \rightarrow C+\gamma$ is not fast enough to occur inside the detector. Then production of $C$ and $D$ would appear as missing energy. In 
particular, light $C, D$ can be produced in the invisible $\Upsilon$ decay

$$
\Upsilon \rightarrow \text { inv }
$$

which is a powerful probe of new physics since its branching ratio in the Standard Model is small, about $10^{-5}$ [16]. In our case, this decay is dominated by the $s$-channel annihilation through the photon, while the $Z$-contribution is suppressed by $m_{\Upsilon}^{4} / m_{Z}^{4}$. We find

$$
\begin{aligned}
\Gamma(\Upsilon \rightarrow C D)= & 2 \alpha \kappa^{2} \cos ^{2} \theta_{W} Q_{d}^{2} \frac{f_{\Upsilon}^{2}}{m_{\Upsilon}} \sqrt{1-2 \frac{M_{C}^{2}+M_{D}^{2}}{m_{\Upsilon}^{2}}+\frac{\left(M_{C}^{2}-M_{D}^{2}\right)^{2}}{m_{\Upsilon}^{4}}} \\
& \times\left[1+\frac{m_{\Upsilon}^{2}}{12}\left(\frac{1}{M_{C}^{2}}+\frac{1}{M_{D}^{2}}\right)\left(1-2 \frac{M_{C}^{2}+M_{D}^{2}}{m_{\Upsilon}^{2}}+\frac{\left(M_{C}^{2}-M_{D}^{2}\right)^{2}}{m_{\Upsilon}^{4}}\right)\right],
\end{aligned}
$$

where $\alpha$ is the fine structure constant, $Q_{d}$ is the down quark charge and $f_{\Upsilon}$ is the $\Upsilon$ decay constant, $\left\langle 0\left|\bar{b} \gamma^{\mu} b\right| \Upsilon\right\rangle=f_{\Upsilon} m_{\Upsilon} \epsilon^{\mu}$ with $\epsilon^{\mu}$ being the $\Upsilon$ polarization vector. In the limit $M_{C, D}^{2} \ll m_{\Upsilon}^{2}$ and $M_{C} \simeq M_{D}=M$, the decay rate becomes

$$
\Gamma(\Upsilon \rightarrow C D) \simeq \frac{1}{3} \alpha \kappa^{2} \cos ^{2} \theta_{W} Q_{d}^{2} \frac{f_{\Upsilon}^{2} m_{\Upsilon}}{M^{2}}
$$

Taking $m_{\Upsilon}(1 S)=9.5 \mathrm{GeV}, \Gamma_{\Upsilon}(1 S)=5.4 \times 10^{-5} \mathrm{GeV}, f_{\Upsilon}=0.7 \mathrm{GeV}$ and using the BaBar limit $\operatorname{BR}(\Upsilon \rightarrow$ inv $)<3 \times 10^{-4}$ at $90 \%$ CL [17], we find

$$
\frac{\kappa}{M}<4 \times 10^{-3} \mathrm{GeV}^{-1} \text {. }
$$

This bound applies to vector boson masses up to a few $\mathrm{GeV}$ and disappears above $m_{\Upsilon} / 2$. An analogous bound from $J / \Psi \rightarrow$ inv is weaker.

We note that the $\Gamma \propto 1 / M^{2}$ dependence is characteristic to production of the longitudinal components of massive vector bosons. The corresponding polarization vector grows with energy as $E / M$, or in other words, at $M \ll m \Upsilon$, the decay is dominated by the Goldstone boson production, whose couplings grow with energy. Thus, stronger constraints on $\kappa$ are expected from the decay of heavier states.

The corresponding bound from the radiative $\Upsilon$ decay $\Upsilon \rightarrow \gamma+$ inv is much weaker. By $C$-parity, such a decay can only be mediated by the $Z$ boson, which brings in the $m_{\Upsilon}^{4} / m_{Z}^{4}$ suppression factor. The resulting constraint is negligible.

\subsection{Invisible $Z$ decay}

The invisible width of the $Z$ boson $\Gamma_{\text {inv }}^{Z}$ is strongly constrained by the LEP measurements [18]. The process $Z \rightarrow C D$ contributes to $\Gamma_{\text {inv }}^{Z}$ for vector boson masses up to about $45 \mathrm{GeV}$, thereby leading to a bound on $\kappa$. We find

$$
\begin{aligned}
\Gamma(Z \rightarrow C D)= & \frac{1}{2 \pi} \kappa^{2} \sin ^{2} \theta_{W} m_{Z} \sqrt{1-2 \frac{M_{C}^{2}+M_{D}^{2}}{m_{Z}^{2}}+\frac{\left(M_{C}^{2}-M_{D}^{2}\right)^{2}}{m_{Z}^{4}}} \\
& \times\left[1+\frac{m_{Z}^{2}}{12}\left(\frac{1}{M_{C}^{2}}+\frac{1}{M_{D}^{2}}\right)\left(1-2 \frac{M_{C}^{2}+M_{D}^{2}}{m_{Z}^{2}}+\frac{\left(M_{C}^{2}-M_{D}^{2}\right)^{2}}{m_{Z}^{4}}\right)\right] .
\end{aligned}
$$


In the limit $M_{C, D}^{2} \ll m_{Z}^{2}$ and $M_{C} \simeq M_{D}=M$, it becomes

$$
\Gamma(Z \rightarrow C D) \simeq \frac{\kappa^{2} \sin ^{2} \theta_{W}}{12 \pi} \frac{m_{Z}^{3}}{M^{2}} .
$$

Taking the bound on the BSM contribution to $\Gamma_{\text {inv }}^{Z}$ to be roughly $3 \mathrm{MeV}$ (twice the experimental error-bar of $\left.\Gamma_{\text {inv }}^{Z}[18]\right)$, we have

$$
\frac{\kappa}{M}<8 \times 10^{-4} \mathrm{GeV}^{-1} .
$$

In the given kinematic range, this constraint is even stronger than the unitarity bound for $\Lambda=1 \mathrm{TeV}$ and comparable to the latter with a multi-TeV cutoff. As explained above, such sensitivity of $Z \rightarrow$ inv to $\kappa$ is due to the $E / M$ enhancement of the longitudinal vector boson production.

\section{4 $B \rightarrow K+$ inv and $K \rightarrow \pi+$ inv}

Flavor changing transitions with missing energy are also a sensitive probe of matter couplings to "invisible" states (see e.g. [19]). The decay $B \rightarrow K+C D$ proceeds via the SM flavor violating $\bar{b} s Z$ and $\bar{b} s \gamma$ vertices with subsequent conversion of $Z, \gamma$ into $C$ and $D$. Numerically, the process is dominated by the $Z$ contribution with the flavor changing vertex $[20,21]$

$$
\mathcal{L}_{\bar{b} s Z}=\lambda_{\bar{b} s Z} \bar{b}_{L} \gamma_{\mu} s_{L} Z^{\mu}
$$

with

$$
\lambda_{\bar{b} s Z}=\frac{g^{3}}{16 \pi^{2} \cos \theta_{W}} V_{t b}^{*} V_{t s} f\left(\frac{m_{t}^{2}}{m_{W}^{2}}\right),
$$

where $V_{i j}$ are the CKM matrix elements and $f(x)$ is the Inami-Lim function [20],

$$
f(x)=\frac{x}{4}\left(\frac{x-6}{x-1}+\frac{3 x+2}{(x-1)^{2}} \ln x\right) .
$$

We find

$$
\begin{aligned}
\Gamma(B \rightarrow K+C D)= & \frac{\kappa^{2} \lambda_{\bar{b} s Z}^{2} \sin ^{2} \theta_{W}}{2^{7} \pi^{3} m_{B}^{3} m_{Z}^{4}} \int_{\left(M_{C}+M_{D}\right)^{2}}^{\left(m_{B}-m_{K}\right)^{2}} \frac{d s}{s} f_{+}^{2}(s) \\
& \times \sqrt{\left(s-M_{C}^{2}-M_{D}^{2}\right)^{2}-4 M_{C}^{2} M_{D}^{2}}\left(\left(s+m_{B}^{2}-m_{K}^{2}\right)^{2}-4 m_{B}^{2} s\right)^{3 / 2} \\
& \times\left[1+\frac{1}{12 s}\left(\frac{1}{M_{C}^{2}}+\frac{1}{M_{D}^{2}}\right)\left(\left(s-M_{C}^{2}-M_{D}^{2}\right)^{2}-4 M_{C}^{2} M_{D}^{2}\right)\right],
\end{aligned}
$$

where the form factor $f_{+}(s)$ is defined by $\left\langle K\left(p_{K}\right)\left|\bar{b} \gamma^{\mu} s\right| B\left(p_{B}\right)\right\rangle=\left(p_{K}+p_{B}\right)^{\mu} f_{+}(s)+\left(p_{B}-\right.$ $\left.p_{K}\right)^{\mu} f_{-}(s)$ with $s=\left(p_{B}-p_{K}\right)^{2}$. The decay rate is dominated by the contribution from large invariant masses of the $C, D$ pair due to the longitudinal vector boson production. This justifies the subleading character of the photon contribution: the corresponding dipole operator can be significant at low invariant masses due to the $1 / \mathrm{s}$ pole, as in the $B \rightarrow \mathrm{Kl}^{+} \mathrm{l}^{-}$ processes (see e.g. [22] for a recent summary). The relative size of various $\Delta F=1$ operators can be found in $[20,21]$, and we find that the photon contribution is unimportant. 


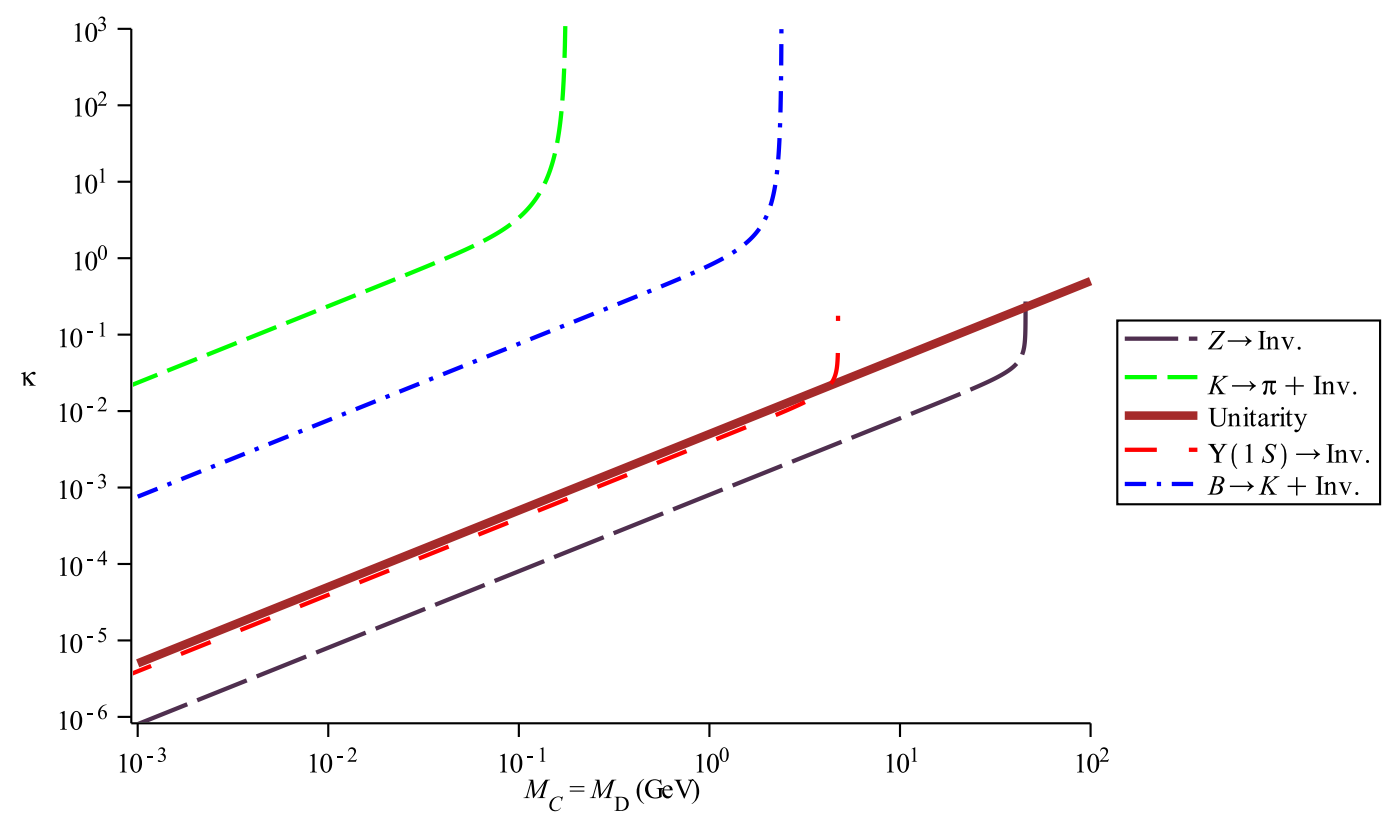

Figure 1. Bounds on $\kappa$. The unitarity bound assumes $\Lambda=1 \mathrm{TeV}$.

The relevant experimental limit has been obtained by $\operatorname{BaBar}: \operatorname{BR}\left(B^{+} \rightarrow K^{+} \nu \bar{\nu}\right)<$ $1.3 \times 10^{-5}$ at $90 \%$ CL [23]. Then taking $f_{+}(0)=0.3$ and using its $s$-dependence from [22], we find

$$
\frac{\kappa}{M}<1 \mathrm{GeV}^{-1}
$$

for $M_{C} \simeq M_{D}=M$ up to roughly $2 \mathrm{GeV}$. The above considerations equally apply to the process $K \rightarrow \pi+$ inv, up to trivial substitutions. We find that the resulting bound is weak, $\kappa / M<30 \mathrm{GeV}^{-1}$. This stems from the $m_{\text {meson }}^{7} /\left(M^{2} m_{Z}^{4}\right)$ behavior of the rate, which favors heavier mesons.

Finally, the Chern-Simons coupling does not contribute to $B \rightarrow C D$ due to the $\epsilon$-tensor contraction, so there is no bound from the $B \rightarrow$ inv decay. Also, $\kappa$ contributes to $(g-2)_{\mu}$ only at the two loop level such that the resulting bound is insignificant.

The summary of the bounds is shown in figure 1 . We see that the most stringent limits are set by the $Z$ invisible width and unitarity considerations. The latter has the advantage of not being limited by kinematics and places a tight bound on $\kappa$ for vector masses up to about $100 \mathrm{GeV}$.

\subsection{Bounds on decaying vector bosons $D \rightarrow C+\gamma$}

When the vector boson mass difference is not too small, the heavier particle, say $D$, will decay inside the detector. In this case, the constraints on $\kappa$ get somewhat modified. The decay width $\Gamma_{D}$ is given by

$$
\Gamma(D \rightarrow C+\gamma)=\frac{\kappa^{2} \cos ^{2} \theta_{W}}{24 \pi} \frac{\left(M_{D}^{2}-M_{C}^{2}\right)^{3}}{M_{D}^{3}}\left(\frac{1}{M_{C}^{2}}+\frac{1}{M_{D}^{2}}\right),
$$


assuming that the $Z$-emission is kinematically forbidden. Given the velocity $v_{D}$ and lifetime $\tau_{D}, D$ decays inside the detector if $v_{D} \tau_{D}=\left|\mathbf{p}_{D}\right| /\left(M_{D} \Gamma_{D}\right)$ is less than the detector size $l_{0}$, which we take to be $\sim 3 \mathrm{~m}$. In this case, $\kappa$ is constrained by radiative decays with missing energy.

Consider the radiative decay $\Upsilon(1 S) \rightarrow \gamma+$ inv. Its branching ratio is constrained by BaBar: $\operatorname{BR}(\Upsilon(1 S) \rightarrow \gamma+$ inv $)<6 \times 10^{-6}$ for a 3-body final state and $M_{C}$ up to about $3 \mathrm{GeV}[24]$. Since $\mathrm{BR}(D \rightarrow C+\gamma) \sim 100 \%$, this requires approximately

$$
\frac{\kappa}{M}<6 \times 10^{-4} \mathrm{GeV}^{-1},
$$

which is the strongest bound on $\kappa$ in the kinematic range $M \lesssim 3 \mathrm{GeV}$. This bound applies for

$$
\Delta M \gtrsim\left(\frac{3 \pi m_{\Upsilon} M}{4 \kappa^{2} \cos ^{2} \theta_{W} l_{0}}\right)^{1 / 3}
$$

where we have made the approximation $M_{D}-M_{C}=\Delta M \ll M \ll m_{\Upsilon}$. For example, taking the maximal allowed $\kappa$ consistent with (3.18) at $M=1 \mathrm{GeV}$, the decay occurs within the detector for $\Delta M>2 \mathrm{MeV}$. (However, since the experimental cut on the photon energy is $150 \mathrm{MeV}, \Delta M$ close to this bound would not lead to a detectable signal.)

On the other hand, the bound on $\kappa$ from the invisible $Z$ width does not change even for decaying $D$. The reason is that the invisible width is defined by subtracting the visible decay width into fermions $\Gamma(Z \rightarrow \bar{f} f)$ from the total width $\Gamma_{Z}$ measured via the energy dependence of the hadronic cross section [18]. Thus, $Z \rightarrow \gamma+$ inv qualifies as "invisible" decay and we still have

$$
\frac{\kappa}{M}<8 \times 10^{-4} \mathrm{GeV}^{-1}
$$

as long as the decay is kinematically allowed.

Finally, the unitarity bound

$$
\frac{\kappa}{M}<\frac{\sqrt{8 \pi}}{\Lambda}
$$

remains intact as well. Another constraint in the higher mass range $m_{Z} / 2 \lesssim M \lesssim 100 \mathrm{GeV}$ is imposed by the LEP monophoton searches $e^{+} e^{-} \rightarrow \gamma+$ inv [25]. We find, however, that it is somewhat weaker than the unitarity bound for $\Lambda=1 \mathrm{TeV}$ (the same applies to $e^{+} e^{-} \rightarrow$ inv).

Thus, the strongest constraints in figure 1 apply also to the case of decaying vector bosons, while the $\Upsilon$ bound becomes competitive and even the tightest one at lower masses. For $M \gtrsim 100 \mathrm{GeV}$, some of the relevant LHC constraints will be discussed in the next section, while their comprehensive analysis requires a separate study.

Let us conclude by remarking on the astrophysical constraints. These apply to very light, up to $\mathcal{O}(\mathrm{MeV})$, particles. In particular, the rate of energy loss in horizontal-branch stars sets stringent bounds on light particle emission in Compton-like scattering $\gamma+e \rightarrow$ $e+C+D$. We find that this cross section in the non-relativistic limit scales approximately as $\alpha^{2} \kappa^{2} /\left(6 \pi m_{e}^{2}\right)(T / M)^{2}$, with $T \sim \mathrm{keV}$ being the core temperature. Comparison to the axion models [26] leads then to the bound $\kappa / M<10^{-7} \mathrm{GeV}^{-1}$ for $M \ll \mathrm{keV}$, which is much stronger than the laboratory constraints in this mass range. Analogous supernova 


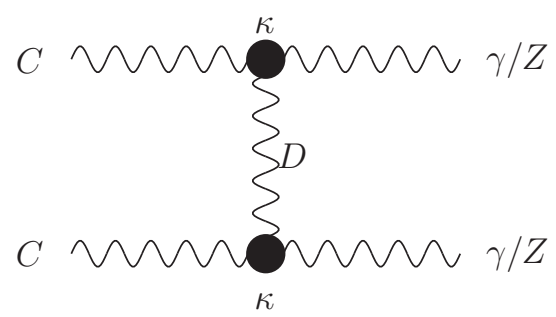

$\langle\sigma v\rangle$

Figure 2. Dark matter annihilation into photons and $Z$-bosons.

cooling considerations extend the range to $\mathcal{O}(\mathrm{MeV})$. A dedicated study of astrophysical constraints will be presented elsewhere.

\section{Vector Dark Matter and the Chern-Simons coupling}

In this section, we consider a special case of the Lagrangian (2.1) with

$$
\delta_{1,2}=0
$$

that is, the new gauge bosons do not mix with the hypercharge. This can be enforced by the $Z_{2}$ symmetry

$$
C_{\mu} \rightarrow-C_{\mu} \quad, \quad D_{\mu} \rightarrow-D_{\mu}
$$

It is straightforward to construct microscopic models which lead to an effective theory endowed with this symmetry at one loop. However, to make the $Z_{2}$ persist at higher loop levels is much more challenging and beyond the scope of this paper.

The relevant Lagrangian in terms of the propagation eigenstates is again given by (2.3), except now $C$ and $D$ do not couple to ordinary matter. The $Z_{2}$ symmetry forbids their kinetic mixing with the photon and the $Z$. This makes the lighter state, $C$, stable and a good dark matter candidate. In what follows, we consider $M_{C}$ of order the electroweak scale such that dark matter is of WIMP type.

Our vector dark matter interacts with the SM only via the Chern-Simons type terms (3.1). These allow for DM annihilation into photons and $Z$ bosons (figure 2 and its cross-version). The corresponding cross sections for $M_{C} \simeq M_{D}=M$ in the non-relativistic 
limit are given by ${ }^{2}$

$$
\begin{aligned}
& \langle\sigma v\rangle(C C \rightarrow \gamma \gamma) \simeq \frac{29 \kappa^{4} \cos ^{4} \theta_{W}}{36 \pi M^{2}}, \\
& \langle\sigma v\rangle(C C \rightarrow \gamma Z) \simeq \frac{\kappa^{4} \sin ^{2} \theta_{W} \cos ^{2} \theta_{W}}{18 \pi M^{2}}\left(1-\frac{M_{Z}^{2}}{4 M^{2}}\right)\left[29-\frac{5 M_{Z}^{2}}{2 M^{2}}+\frac{5 M_{Z}^{4}}{16 M^{4}}\right] \Theta\left(2 M-M_{Z}\right), \\
& \langle\sigma v\rangle(C C \rightarrow Z Z) \simeq \frac{\kappa^{4} \sin ^{4} \theta_{W}}{36 \pi M^{2}} \sqrt{1-\frac{M_{Z}^{2}}{M^{2}}}\left(1-\frac{M_{Z}^{2}}{2 M^{2}}\right)^{-2}\left[29-34 \frac{M_{Z}^{2}}{M^{2}}+14 \frac{M_{Z}^{4}}{M^{4}}\right] \Theta\left(M-M_{Z}\right),
\end{aligned}
$$

where $\Theta$ is the Heaviside distribution. These processes both regulate dark matter abundance and lead to potentially observable gamma-ray signatures, which we study in detail below.

The distinctive feature of the model is the presence of monochromatic gamma-ray lines in the spectrum of photons coming from the Galactic Center (see e.g. [27]). In particular, for heavy dark matter $\left(M^{2} \gg M_{Z}^{2}\right)$, the final states $\gamma \gamma, \gamma Z$ and $Z Z$ are produced in the proportion $\cos ^{4} \theta_{W}, 2 \sin ^{2} \theta_{W} \cos ^{2} \theta_{W}$ and $\sin ^{4} \theta_{W}$, respectively. This implies that continuous gamma-ray emission is subdominant and constitutes about a third of the annihilation cross section, while the monochromatic gamma-ray emission dominates.

\subsection{WMAP/PLANCK constraints}

Assuming that dark matter is thermally produced, its abundance should be consistent with the WIMP freeze-out paradigm. As explained above, the only DM annihilation channel is $C C \rightarrow V V$ with $V=\gamma, Z$. The corresponding cross section must be in a rather narrow window to fit observations. The left panel of figure 3 shows parameter space consistent with the WMAP/PLANCK measurements $[28,29]$ of the DM relic abundance for different values of $\kappa, M_{C}$ and $M_{D}$. For generality, we allow for vastly different $M_{C}$ and $M_{D}$ in our numerical analysis. In the case $M_{C}^{2} \ll M_{D}^{2}$, the scaling behaviour $\langle\sigma v\rangle \sim \kappa^{4} / M^{2}$ of eq. (4.3) is replaced by

$$
\langle\sigma v\rangle \sim \kappa^{4} \frac{M_{C}^{2}}{M_{D}^{4}}
$$

which stems from the momentum factors at the vertices. Thus, the annihilation cross section grows with the dark matter mass and, in turn, the WMAP/PLANCK-allowed $\kappa$ 's decrease with increasing $M_{C}$. The former take on rather natural values of order one for $M_{D}$ between $100 \mathrm{GeV}$ and several $\mathrm{TeV}$. The main annihilation channel is $C C \rightarrow \gamma \gamma$, which for $M_{C} \simeq M_{D} \simeq 200 \mathrm{GeV}$ constitutes about $60 \%$ of the total cross section. The channels $C C \rightarrow \gamma Z$ and $C C \rightarrow Z Z$ contribute $35 \%$ and $5 \%$, respectively. The allowed parameter space is subject to the FERMI and HESS constraints on the gamma-ray emission, which we study in the next subsection. 
$\kappa$

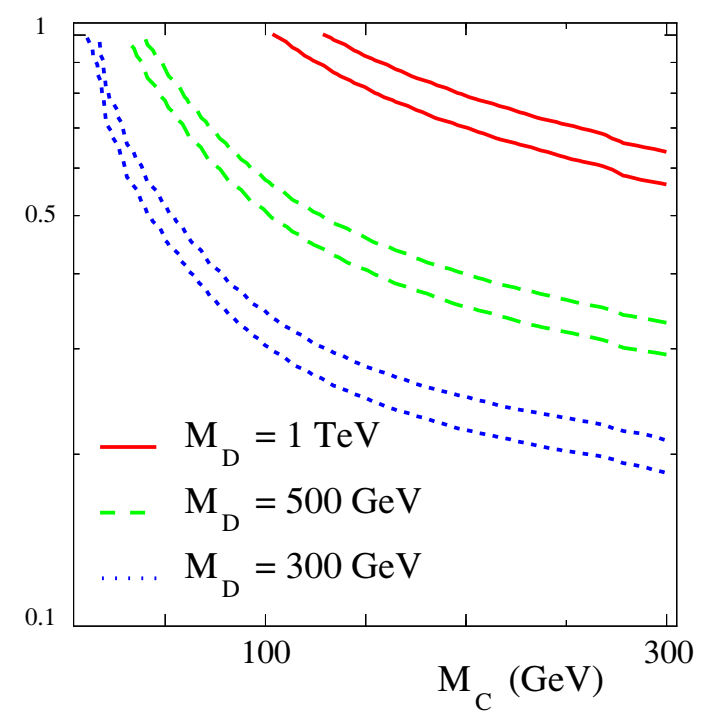

$M_{D}(\mathrm{GeV})$

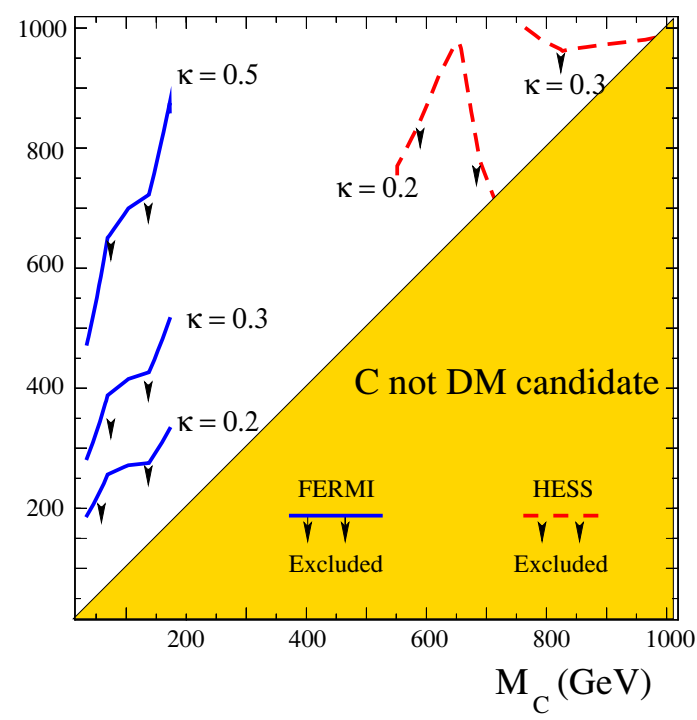

Figure 3. Left: the areas between the lines represent values of $\kappa$ consistent with the WMAP/PLANCK constraint as a function of $M_{C}$ for different values of $M_{D}: 300 \mathrm{GeV}$ (dotted blue), $500 \mathrm{GeV}$ (dashed green), and $1 \mathrm{TeV}$ (solid red). Right: constraints from the FERMI and HESS searches for monochromatic gamma-ray lines in the plane $\left(M_{C}, M_{D}\right)$. (The area below the curve for a given $\kappa$ is excluded.)
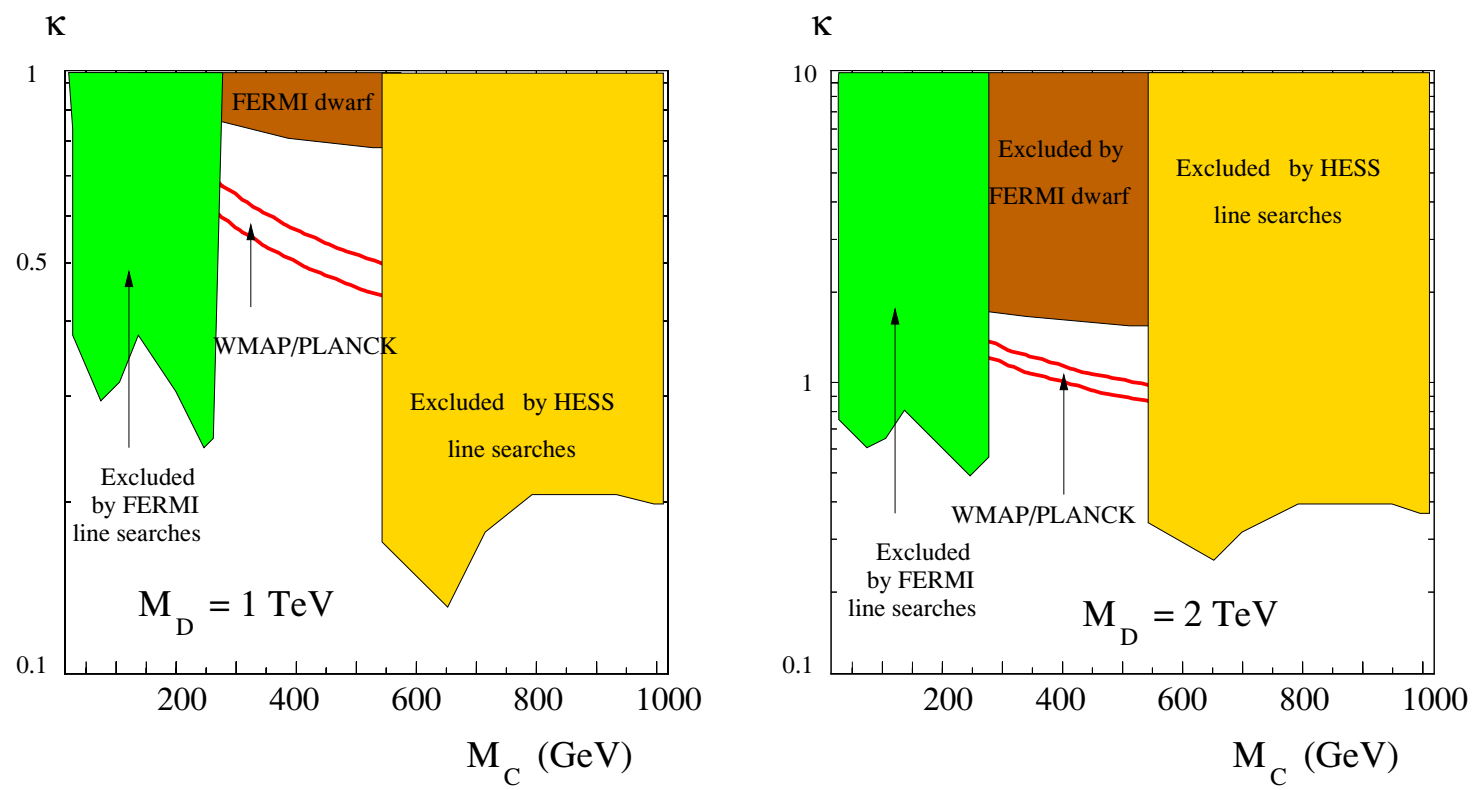

Figure 4. FERMI and HESS constraints on gamma-ray monochromatic lines and continuum in the plane $\left(M_{C}, \kappa\right)$ for $M_{D}=1 \mathrm{TeV}$ [left] and $2 \mathrm{TeV}$ [right]. The area between the red lines is consistent with thermal DM relic abundance. 


\subsection{Indirect DM detection constraints}

Dark matter can be detected indirectly by observing products of its annihilation in regions with enhanced DM density. The main feature of the Chern-Simons-type dark matter is that the dominant annihilation channel leads to a di-photon final state. These photons are monochromatic due to the low DM velocity nowadays $\left(v_{C} \simeq 300 \mathrm{kms}^{-1}\right)$, which is a "smoking-gun" signature of our model. The proportion of the di-photon final state increases somewhat compared to that in the Early Universe due to the (slight) reduction of the centerof-mass energy of the colliding DM particles. In particular, for $M_{C} \simeq M_{D} \simeq 200 \mathrm{GeV}$, the channels $C C \rightarrow \gamma \gamma, C C \rightarrow \gamma Z$ and $C C \rightarrow Z Z$ constitute approximately $63 \%, 33 \%, 4 \%$ of the total cross section. One therefore expects an intense monochromatic gamma-ray line at $E_{\gamma}=M_{C}$ and a weaker line at $E_{\gamma}=M_{C}-M_{Z}^{2} /\left(4 M_{C}\right)$. Such lines would provide convincing evidence for DM annihilation since astrophysical processes are very unlikely to generate such a photon spectrum.

Recently, FERMI [30-32] and HESS [33] collaborations have released their analyses of the monochromatic line searches around the Galactic Center. Due to its limited energy sensitivity, the FERMI satellite sets a bound on the di-photon annihilation cross section $\langle\sigma v\rangle_{\gamma \gamma}$ in the DM mass range $1 \mathrm{GeV} \lesssim M_{C} \lesssim 300 \mathrm{GeV}$. HESS, on the other hand, is restrained by its threshold limitations and provides bounds in the DM mass range $500 \mathrm{GeV} \lesssim M_{C} \lesssim 20 \mathrm{TeV}^{3}$ Combining the two analyses allows us to eliminate large portions of parameter space as shown in figure 3 [right] and figure 4 . We note that increasing the mediator mass $M_{D}$ has the same effect as decreasing the coupling $\kappa$. The important conclusion is that FERMI and HESS exclude the possibility of thermal DM relic abundance in the relevant mass ranges. Indeed, their bounds are of order $\langle\sigma v\rangle_{\gamma \gamma} \lesssim 10^{-27} \mathrm{~cm}^{3} \mathrm{~s}^{-1}$, whereas thermal dark matter requires $\langle\sigma v\rangle \simeq 10^{-26} \mathrm{~cm}^{3} \mathrm{~s}^{-1}$.

To fill the gap between 300 and $500 \mathrm{GeV}$ where the monochromatic signal is not constrained, one can use the diffuse gamma-ray flux. Indeed, even though the FERMI energy cuf-off is at $300 \mathrm{GeV}$, annihilation of heavy particles produces a continuum photon spectrum which can be detected by FERMI. In our case, the continuum comes from the $Z Z$ and $Z \gamma$ final states with subsequent $Z$-decay. Since such final states contribute about $40 \%$ to the total cross section, the resulting constraint is not very strong. There exist several analyses of bounds on DM annihilation in the galactic halo [34], galactic center [35] and dwarf galaxies [36, 37]. The latter provides the strongest FERMI constraint at the moment, while that from HESS is very weak, and we use it to restrict our parameter space (figure 4). The conclusion is that thermal DM in the $300-500 \mathrm{GeV}$ mass range remains viable and can soon be tested by HESS/FERMI.

\footnotetext{
${ }^{2}$ For simplicity, we have assumed a single mass scale for the vectors with $D$ being somewhat heavier such that it decays into $C$ and a photon. Further details are unimportant for our purposes. However, we do not consider (almost exactly) degenerate $C$ and $D$, which would require an additional symmetry justification and inclusion of the coannihilation channel in the relic abundance calculation.

${ }^{3}$ HESS reports its results for the Einasto DM distribution profile, while FERMI has extended its study to other profiles as well. To be conservative, we use the FERMI limits for the isothermal profile.
} 


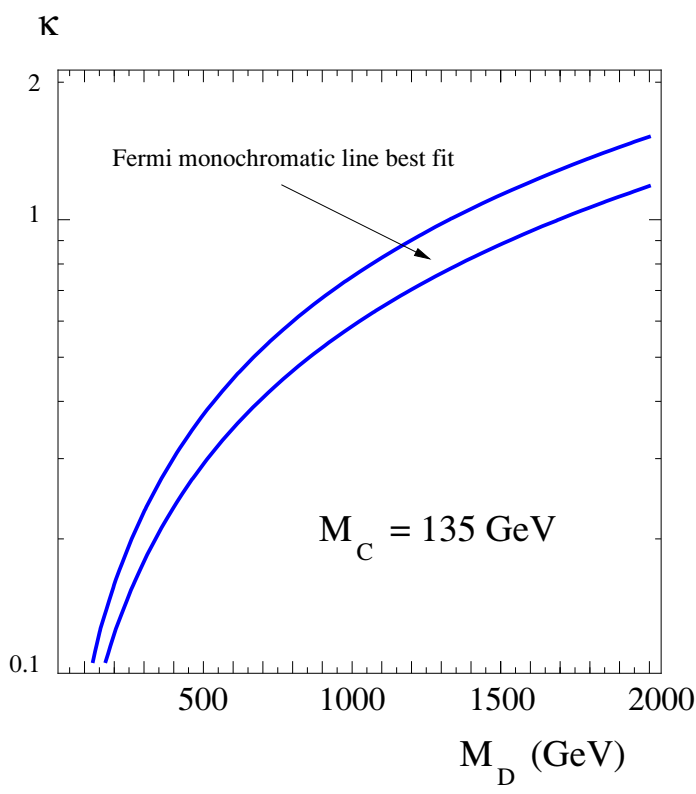

Figure 5. Parameter space (between the lines) satisfying $\langle\sigma v\rangle_{\gamma \gamma}=\left(1.27 \pm 0.32_{-0.28}^{+0.18}\right) \times$ $10^{-27} \mathrm{~cm}^{3} \mathrm{~s}^{-1}$ and fitting the tentative FERMI gamma-ray line at $135 \mathrm{GeV}$.

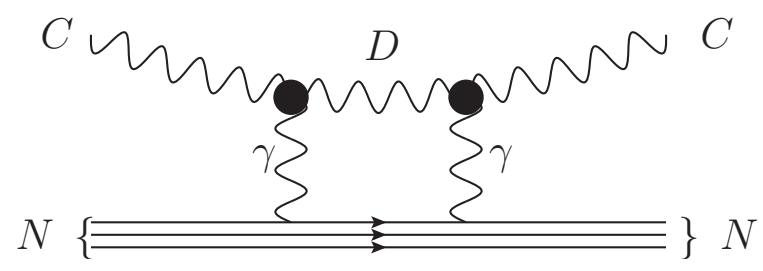

Figure 6. Dark matter scattering off a nucleon.

\subsection{On the tentative $135 \mathrm{GeV}$ gamma-ray line}

When analyzing FERMI data, several groups found some indications of a monochromatic $(135 \mathrm{GeV})$ gamma-ray line from the galactic center [38-40]. The significance of the "signal" appears to be around 3.3 sigma taking into account the look-elsewhere effect, although this has not been confirmed by the FERMI collaboration. A somewhat optimistic interpretation of the line is that it could be due to DM annihilation at the galactic center (see [41-44] for recent discussions), with the cross section $\langle\sigma v\rangle_{\gamma \gamma}=\left(1.27 \pm 0.32_{-0.28}^{+0.18}\right) \times 10^{-27} \mathrm{~cm}^{3} \mathrm{~s}^{-1}$ for an Einasto-like profile [38, 39].

In this work, we will be impartial as to whether the line is really present in the data or not. Instead, we use the analysis of $[38,39]$ as an example to show that the hypercharge portal can easily accommodate a monochromatic signal from the sky. Our result is shown in figure 5. Having fixed $M_{C}=135 \mathrm{GeV}$, we observe that the gamma-ray line can be accommodated for any mediator mass $M_{D}$. As explained above, the continuum constraint is inefficient here since it applies to subdominant final states. On the other hand, the required annihilation cross section is too small for DM to be a thermal relic. 


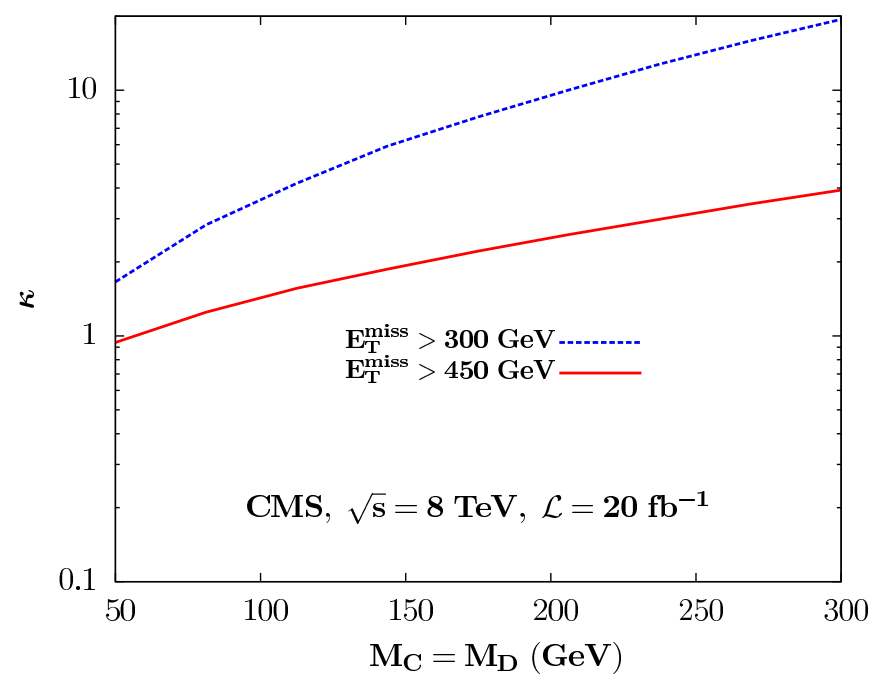

Figure 7. Limit on $\kappa$ from monojet searches at CMS for $\sqrt{s}=8 \mathrm{TeV}$ and $20 \mathrm{fb}^{-1}$ integrated luminosity.

\subsection{Direct detection constraints}

An important constraint on properties of dark matter is set by direct detection experiments which utilize possible DM interactions with nuclei. In our case, dark matter scattering off nuclei is described by the 1-loop diagram of figure 6 together with its cross-version, and similar diagrams with $Z$-bosons in the loop. Setting for simplicity $M_{C} \simeq M_{D}=M$, we find that in the non-relativistic limit this process is described by the operators

$$
\begin{aligned}
O_{S I} & \sim \frac{\alpha \kappa^{2}}{4 \pi} \frac{m_{N}}{M^{2}} \bar{\Psi} \Psi C^{\mu} C_{\mu}, \\
O_{S D} & \sim \frac{\alpha \kappa^{2}}{4 \pi} \frac{1}{M^{2}} \epsilon_{\mu \nu \rho \sigma} \bar{\Psi} \gamma^{\mu} \gamma^{5} \Psi C^{\nu} i \partial^{\rho} C^{\sigma},
\end{aligned}
$$

where $m_{N}$ is a hadronic scale of the order of the nucleon mass and $\Psi$ is the nucleon spinor. $O_{S I}$ and $O_{S D}$ are responsible for spin-independent and spin-dependent scattering, respectively. The former is suppressed both by the loop factor and the nucleon mass, while the latter is suppressed by the loop factor only. The resulting cross sections are quite small, $\sigma_{S I} \sim \kappa^{4} / M^{2}(\alpha / 4 \pi)^{2}\left(m_{N} / M\right)^{4} \sim 10^{-46} \mathrm{~cm}^{2}$ for $\kappa \sim 1$ and $M \sim 100 \mathrm{GeV}$, whereas the spin-dependent cross-section is of the order of $\sigma_{S D} \sim \kappa^{4} / M^{2}(\alpha / 4 \pi)^{2}\left(m_{N} / M\right)^{2} \sim 10^{-42} \mathrm{~cm}^{2}$ for the same parameters. The current XENON100 bounds are $\sigma_{S I} \lesssim \mathcal{O}\left(10^{-45}\right) \mathrm{cm}^{2}[45]$ and $\sigma_{S D} \lesssim \mathcal{O}\left(10^{-40}\right) \mathrm{cm}^{2}$ [46] for the DM mass around $100 \mathrm{GeV}$ (which maximizes the XENON100 sensitivity). We thus conclude that no significant bounds on $\kappa$ can be obtained from direct detection experiments. Furthermore, since the gamma-ray constraints require $\kappa<\mathcal{O}\left(10^{-1}\right)$ in this mass range, the prospects for direct DM detection are rather bleak, orders of magnitude beyond the projected sensitivity of XENON1T [47].

\subsection{LHC monojet constraints}

The vector states $C$ and $D$ can be produced at the LHC. If their mass difference is not sufficiently large, the photon coming from $D$-decay would not pass the experimental cut 
on the photon energy $\left(p_{T}>150 \mathrm{GeV}\right)$. In this case, production of $C$ and $D$ would appear as missing energy. The latter can be detected in conjunction with a jet coming from initial-state radiation, which sets a bound on DM production (see also [48]).

In this subsection, we estimate the sensitivity of current monojet searches at the LHC to dark matter production through its coupling to $Z$ and $\gamma$. Our constraints are based on the search for monojets performed by the CMS collaboration which makes use of $19.5 \mathrm{fb}^{-1}$ of data at $8 \mathrm{TeV}$ center of mass energy [49]. The basic selection requirements used by the CMS experiment for monojet events are as follows:

- at least 1 jet with $p_{T}^{j}>110 \mathrm{GeV}$ and $\left|\eta^{j}\right|<2.4$;

- at most 2 jets with $p_{T}^{j}>30 \mathrm{GeV}$;

- no isolated leptons.

The CMS collaboration quotes the event yields for 7 different cuts on the missing transverse momentum $p_{T}^{\text {miss }}$ between 250 and $550 \mathrm{GeV}$. These are largely dominated by the SM backgrounds, namely $Z+$ jets, where the $Z$ boson decays invisibly, and $W+$ jets, where the $W$ boson decays leptonically and the charged lepton is not reconstructed. In particular, with $19.5 \mathrm{fb}^{-1}$ data, the CMS collaboration estimates the background to be $18506 \pm 690(1931 \pm 131)$ events for $p_{T}^{\text {miss }}>300(450) \mathrm{GeV}$.

A virtual $Z$-boson or a photon produced with a significant transverse momentum and coupled to invisible states can also lead to the topology that is targeted by the monojet searches. In order to estimate the sensitivity of the CMS monojet search to the " $Z / \gamma \rightarrow$ invisible" signal, we generate the $p p \rightarrow Z / \gamma+$ jets $\rightarrow \mathrm{CD}+$ jets process at the parton level with Madgraph 5 [50]. Showering and hadronization is performed using Pythia 6 [51], while Delphes 1.9 [52] is employed to simulate the ATLAS and CMS detector response. We have imposed the analysis cuts listed above on the simulated events to find the signal efficiency. As a cross-check, we have passed $(Z \rightarrow \nu \nu)+$ jets background events through the same simulation chain, obtaining efficiencies consistent with the data-driven estimates of that background provided by CMS.

We use the total event cross section to put constraints on the dark matter coupling to the $Z / \gamma$ gauge bosons. We compute the observed $95 \% \mathrm{CL}$ exclusion limits on the dark matter-SM coupling $\kappa$ for given masses $M_{C}, M_{D}$ by requiring (see, e.g. [53])

$$
\chi^{2}=\frac{\left(N_{o b s}-N_{S M}-N_{D M}\left(M_{C}, M_{D}, \kappa\right)\right)^{2}}{N_{S M}+N_{D M}\left(M_{C}, M_{D}, \kappa\right)+\sigma_{S M}^{2}}=3.84 .
$$

Here $N_{o b s}$ is the number of observed events, $N_{S M}$ the number of expected events, $N_{D M}$ the number of expected signal events and $\sigma_{S M}$ being the uncertainty in the predicted number of backgrounds events. The expected strongest bounds should come from the analysis with the hardest $p_{T}^{\text {miss }}>550 \mathrm{GeV}$ cuts, but the strongest observed bound come from the $p_{T}^{\text {miss }}>450 \mathrm{GeV}$ cuts due to an important downward fluctuations in the data. Figure 7 shows the resulting limits on $\kappa$ for two different sets of cuts, $p_{T}^{\text {miss }}>300 \mathrm{GeV}$ and $p_{T}^{\text {miss }}>450 \mathrm{GeV}$, with the latter providing the best limit. We see that the current monojet bounds are relatively weak, $\kappa<\mathcal{O}(1)$ for $M_{C} \sim M_{D} \sim 100 \mathrm{GeV}$, and not competetive with the constraints from the monochromatic gamma-ray searches. 


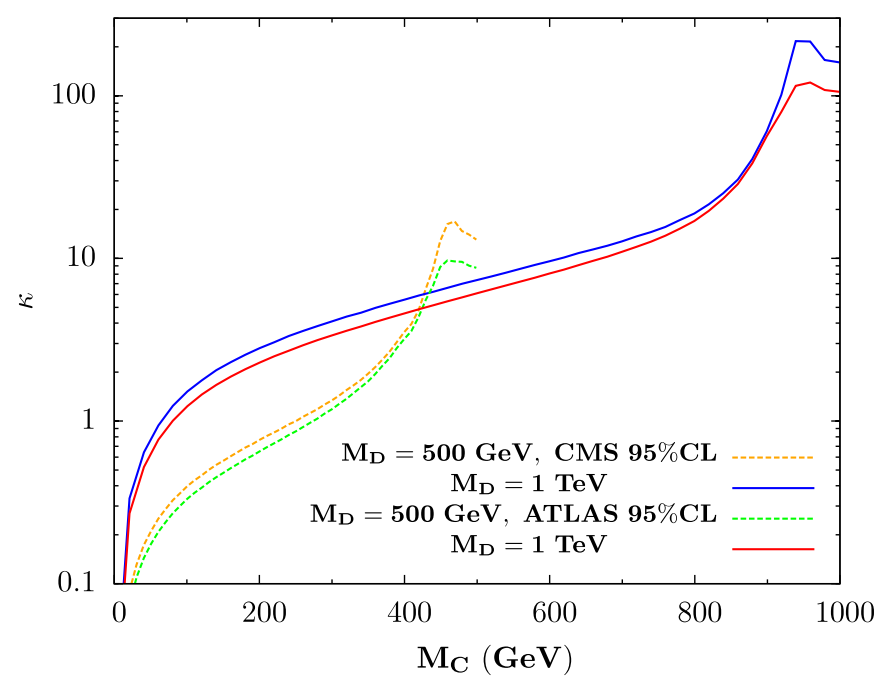

Figure 8. Limits on $\kappa$ from monophoton searches at $\mathrm{CMS}\left(5 \mathrm{fb}^{-1}\right.$ at $\left.\sqrt{s}=7 \mathrm{TeV}\right)$ and ATLAS $\left(4.6 \mathrm{fb}^{-1}\right.$ at $\left.\sqrt{s}=7 \mathrm{TeV}\right)$.

\subsection{LHC monophoton constraints}

Another characteristic collider signature of vector DM production is monophoton emission plus missing energy. In this case, $C$ and $D$ are produced on-shell through the photon or $Z$, while their mass difference must be sufficiently large such that $D$ decays inside the detector and the photon energy is above the threshold. ${ }^{4}$ We rely on the search for a single photon performed by the CMS collaboration which makes use of $5 \mathrm{fb}^{-1}$ of data at $7 \mathrm{TeV}$ center of mass energy [54] and the one performed by the ATLAS collaboration which makes use of $4.6 \mathrm{fb}^{-1}$ of data at $7 \mathrm{TeV}$ center of mass energy [55]. The basic selection requirements used by the CMS experiment for monophoton events are as follows:

- 1 photon with $p_{T}^{\gamma}>145 \mathrm{GeV}$ and $\left|\eta^{\gamma}\right|<1.44$;

- $p_{T}^{\text {miss }}>130 \mathrm{GeV}$;

- no jet with $p_{T}^{j}>20 \mathrm{GeV}$ that is $\Delta R>0.04$ away from the photon candidate; ${ }^{5}$

- no jet with $p_{T}^{j}>40 \mathrm{GeV}$ and $\left|\eta^{j}\right|<3.0$ within $\Delta R<0.5$ of the axis of the photon;

Analogous requirements used by ATLAS are:

- 1 photon with $p_{T}^{\gamma}>150 \mathrm{GeV}$ and $\left|\eta^{\gamma}\right|<2.37$;

- $p_{T}^{\text {miss }}>150 \mathrm{GeV}$;

\footnotetext{
${ }^{4}$ Note that the monophoton topology could also appear in the scenario considered in the previous subsection, where both $C$ and $D$ escape detection, with the photon originating in initial-state radiation. However, the corresponding constraints are superseded by those driven by the monojet searches, so that we will not pay more attention to this case.

${ }^{5}$ Note that this cut originally applies on a track. Yet its conversion into a cut on jets, related to the implementation in Delphes, makes it superfluous in consideration of the other conditions applied.
} 
- no more than 1 jet with $p_{T}^{j}>30 \mathrm{GeV}$ and $\left|\eta^{j}\right|<4.5$;

- $\Delta \Phi\left(\gamma, p_{T}^{\text {miss }}\right)>0.4, \Delta R(\gamma, j e t)>0.4$ and $\Delta \Phi\left(j e t, p_{T}^{\text {miss }}\right)>0.4$;

The event yields obtained by ATLAS and CMS are largely dominated by the SM backgrounds, namely $Z+\gamma$, where the $Z$ boson decays invisibly, and $W+\gamma$, where the $W$ boson decays leptonically and the charged lepton is not reconstructed. Since ATLAS accepts events with one jet, $W / Z+$ jets is also an important background for the ATLAS analysis. With $4.6 \mathrm{fb}^{-1}$ data, the ATLAS collaboration estimates the background to be $137 \pm 18$ (stat. $) \pm 9$ (syst.) events and observed 116 events. The analogous numbers for CMS with $5 \mathrm{fb}^{-1}$ are $75.1 \pm 9.4$ and 73 events, respectively.

In order to estimate the sensitivity of the ATLAS and CMS single photon search to DM production, we have generated the $p p \rightarrow Z / \gamma \rightarrow C D \rightarrow C C+\gamma$ process. We have used the program Madgraph 5 [50] for the channels at the parton level. Showering and hadronisation was performed using Pythia 6 [51] and Delphes 1.9 [52] was employed to simulate the CMS detector response. We have imposed the analysis cuts listed above on the simulated events to find the signal efficiency and used the total event cross-section to constrain the DM coupling to $\gamma$ and $Z$. The observed 95\%CL exclusion limits on $\kappa$ for given $M_{C}, M_{D}$ are obtained by requiring

$$
\chi^{2}=\frac{\left(N_{o b s}-N_{S M}-N_{D M}\left(M_{C}, M_{D}, \kappa\right)\right)^{2}}{N_{S M}+N_{D M}\left(M_{C}, M_{D}, \kappa\right)+\sigma_{S M}^{2}}=3.84 .
$$

The resulting limits on $\kappa$ for two choices of $M_{D}=500 \mathrm{GeV}$ and $M_{D}=1 \mathrm{TeV}$ are shown in figure 8 . In the latter case, the bounds are relatively weak, $\kappa<1$ for $M_{C}>$ $100 \mathrm{GeV}$, and do not constrain the parameter space consistent with WMAP/PLANCK, FERMI and HESS (figure 4). For $M_{D}=500 \mathrm{GeV}$, the monophoton constraint is more important, although it does not yet probe interesting regions of parameter space (figure 3 ). In particular, it does not rule out the DM interpretation of the $135 \mathrm{GeV}$ gamma-ray line (figure 5). Indeed, for $M_{C}=135 \mathrm{GeV}$, the LHC bound is about $\kappa<0.5$, whereas the gamma-ray line requires $\kappa \sim 0.3$.

We thus find that the monophoton constraint is not yet competitive with the astrophysical/cosmological ones. We have also checked that no useful constraint is imposed by searches for mono- $Z$ emission $(D \rightarrow Z+C)$, mostly due to its smaller production cross section.

\subsection{Summary of constraints}

For the DM mass above $100 \mathrm{GeV}$, the most relevant laboratory constraints are imposed by the LHC searches for monojets and monophotons. The former are applicable for quasidegenerate $C$ and $D$, while the latter apply if there is a substantial mass difference between them. The monophoton constraint is rather tight for light DM, e.g. $\kappa<$ few $\times 10^{-1}$ for $M_{C} \sim$ $100 \mathrm{GeV}$ and $M_{D} \sim 500 \mathrm{GeV}$. This is stronger than the unitarity bound (3.4), which only applies for $\Lambda \gg M_{C, D}$. On the other hand, the monojet constraint is rather weak, $\kappa \lesssim 1$.

The most important bounds on the model are imposed by astrophysical observations, in particular, by FERMI and HESS searches for monochromatic gamma-ray lines. These 
exclude substantial regions of parameter space even for relatively heavy dark matter, $M_{C, D} \sim 1 \mathrm{TeV}$. Analogous bounds from continuum gamma-ray emission are significantly weaker as the latter is subleading in our framework (unlike in other models [56]), while direct DM detection is inefficient due to loop suppression. These constraints still allow for thermal DM in the mass range $200-600 \mathrm{GeV}$ (figure 4).

Finally, the model allows for an "optimistic" interpretation of the tentative $135 \mathrm{GeV}$ gamma-ray line in the FERMI data. The line can be due to (non-thermal) dark matter annihilation with $M_{C} \simeq 135 \mathrm{GeV}$ for a range of the mediator mass $M_{D}$. This interpretation is consistent with the constraints coming from the continuum gamma-ray emission, direct DM detection and the LHC searches.

\section{Conclusion}

We have considered the possibility that the hidden sector contains more than one massive vector fields. In this case, an additional dim-4 interaction structure of the Chern-Simons type becomes possible. It couples the hypercharge field strength to the antisymmetric combination of the massive vectors. If the latter are long-lived, the phenomenological signatures of such a coupling include missing energy in decays of various mesons and $Z$, as well as monojet and monophoton production at the LHC.

The hidden sector may possess a $Z_{2}$ symmetry, which would make the lighter vector field stable and a good dark matter candidate. The characteristic signature of this scenario is monochromatic gamma-ray emission from the Galactic Center, while the corresponding continuum contribution is suppressed. We find that this possibility is consistent with other constraints, including those from the LHC and direct DM detection. Large portions of the allowed parameter space can be probed both by indirect DM detection and the LHC monophoton searches.

\section{Acknowledgments}

We are grateful to A. Ali for useful discussions. This work was partially supported by the French ANR TAPDMS ANR-09-JCJC-0146, the Spanish MICINNs Consolider-Ingenio 2010 Programme under grant Multi- Dark CSD2009-00064 and the Collaborative Research Center SFB 676 of the DFG, "Particles, Strings, and the Early Universe". Y. M. and J. Q. also acknowledge partial support from the European Union FP7 ITN INVISIBLES (Marie Curie Actions, PITN- GA-2011- 289442) and the ERC advanced grant Higgs@LHC.

Open Access. This article is distributed under the terms of the Creative Commons Attribution License which permits any use, distribution and reproduction in any medium, provided the original author(s) and source are credited.

\section{References}

[1] D.J. Gross, J.A. Harvey, E.J. Martinec and R. Rohm, The Heterotic String, Phys. Rev. Lett. 54 (1985) 502 [INSPIRE]. 
[2] W. Buchmüller, K. Hamaguchi, O. Lebedev and M. Ratz, Supersymmetric standard model from the heterotic string, Phys. Rev. Lett. 96 (2006) 121602 [hep-ph/0511035] [INSPIRE].

[3] B. Patt and F. Wilczek, Higgs-field portal into hidden sectors, hep-ph/0605188 [INSPIRE].

[4] T. Asaka, S. Blanchet and M. Shaposhnikov, The nuMSM, dark matter and neutrino masses, Phys. Lett. B 631 (2005) 151 [hep-ph/0503065] [INSPIRE].

[5] V. Silveira and A. Zee, SCALAR PHANTOMS, Phys. Lett. B 161 (1985) 136 [inSPIRE].

[6] O. Lebedev, H.M. Lee and Y. Mambrini, Vector Higgs-portal dark matter and the invisible Higgs, Phys. Lett. B 707 (2012) 570 [arXiv:1111.4482] [INSPIRE].

[7] O. Lebedev and A. Westphal, Metastable Electroweak Vacuum: Implications for Inflation, Phys. Lett. B 719 (2013) 415 [arXiv: 1210.6987] [INSPIRE].

[8] C. Corianò, N. Irges and E. Kiritsis, On the effective theory of low scale orientifold string vacua, Nucl. Phys. B 746 (2006) 77 [hep-ph/0510332] [INSPIRE].

[9] P. Anastasopoulos, M. Bianchi, E. Dudas and E. Kiritsis, Anomalies, anomalous U(1)'s and generalized Chern-Simons terms, JHEP 11 (2006) 057 [hep-th/0605225] [INSPIRE].

[10] I. Antoniadis, A. Boyarsky, S. Espahbodi, O. Ruchayskiy and J.D. Wells, Anomaly driven signatures of new invisible physics at the Large Hadron Collider,

Nucl. Phys. B 824 (2010) 296 [arXiv:0901.0639] [INSPIRE].

[11] E. Dudas, Y. Mambrini, S. Pokorski and A. Romagnoni, (In)visible Z-prime and dark matter, JHEP 08 (2009) 014 [arXiv: 0904.1745] [INSPIRE].

[12] E. Dudas, Y. Mambrini, S. Pokorski and A. Romagnoni, Extra U(1) as natural source of a monochromatic gamma ray line, JHEP 10 (2012) 123 [arXiv:1205.1520] [INSPIRE].

[13] I. Antoniadis, Motivation for weakly interacting SubeV particles, DESY-PROC-2010-03.

[14] Y. Farzan and A.R. Akbarieh, Natural explanation for $130 \mathrm{GeV}$ photon line within vector boson dark matter model, arXiv:1211.4685 [INSPIRE].

[15] B. Holdom, Two U(1)'s and Epsilon Charge Shifts, Phys. Lett. B 166 (1986) 196 [inSPIRE].

[16] L. Chang, O. Lebedev and J. Ng, On the invisible decays of the Upsilon and J/Psi resonances, Phys. Lett. B 441 (1998) 419 [hep-ph/9806487] [INSPIRE].

[17] BaBAR collaboration, B. Aubert et al., A Search for Invisible Decays of the Upsilon(1S), Phys. Rev. Lett. 103 (2009) 251801 [arXiv:0908.2840] [INSPIRE].

[18] ALEPH, DELPHI, L3, OPAL, SLD collaboration, LEP Electroweak Working Group, SLD Electroweak Group, SLD Heavy Flavour Group, S. Schael et al., Precision electroweak measurements on the $Z$ resonance, Phys. Rept. 427 (2006) 257 [hep-ex/0509008] [INSPIRE].

[19] S. Andreas, O. Lebedev, S. Ramos-Sanchez and A. Ringwald, Constraints on a very light CP-odd Higgs of the NMSSM and other axion-like particles, JHEP 08 (2010) 003 [arXiv: 1005.3978] [INSPIRE].

[20] T. Inami and C. Lim, Effects of Superheavy Quarks and Leptons in Low-Energy Weak Processes $k(L) \rightarrow \mu \bar{\mu}, K^{+} \rightarrow \pi^{+}$Neutrino anti-neutrino and $K^{0} \rightarrow \bar{K}^{0}$,

Prog. Theor. Phys. 65 (1981) 297 [Erratum ibid. 65 (1981) 1772] [INSPIRE].

[21] B. Grinstein, M.J. Savage and M.B. Wise, $B \rightarrow X_{s} e^{+} e^{-}$in the Six Quark Model, Nucl. Phys. B 319 (1989) 271 [inSPIRE]. 
[22] G. Buchalla, Precision flavour physics with $B \rightarrow K \nu \bar{\nu}$ and $B \rightarrow K l^{+} l^{-}$, Nucl. Phys. Proc. Suppl. 209 (2010) 137 [arXiv: 1010.2674] [InSPIRE].

[23] BaBar collaboration, P. del Amo Sanchez et al., Search for the Rare Decay B $\rightarrow K \nu \bar{u}$, Phys. Rev. D 82 (2010) 112002 [arXiv:1009.1529] [InSPIRE].

[24] BaBar collaboration, P. del Amo Sanchez et al., Search for Production of Invisible Final States in Single-Photon Decays of $\Upsilon(1 S)$, Phys. Rev. Lett. 107 (2011) 021804 [arXiv: 1007.4646] [INSPIRE].

[25] L3 collaboration, P. Achard et al., Single photon and multiphoton events with missing energy in $e^{+} e^{-}$collisions at LEP, Phys. Lett. B 587 (2004) 16 [hep-ex/0402002] [INSPIRE].

[26] G. Raffelt and A. Weiss, Red giant bound on the axion - electron coupling revisited, Phys. Rev. D 51 (1995) 1495 [hep-ph/9410205] [INSPIRE].

[27] G. Vertongen and C. Weniger, Hunting Dark Matter Gamma-Ray Lines with the Fermi LAT, JCAP 05 (2011) 027 [arXiv: 1101.2610] [INSPIRE].

[28] N. Jarosik et al., Seven-Year Wilkinson Microwave Anisotropy Probe (WMAP) Observations: Sky Maps, Systematic Errors and Basic Results, Astrophys. J. Suppl. 192 (2011) 14 [arXiv: 1001.4744] [INSPIRE].

[29] Planck collaboration, Planck 2013 results. I. Overview of products and scientific results, arXiv: 1303.5062 [INSPIRE].

[30] LAT collaboration, M. Ackermann et al., Fermi LAT Search for Dark Matter in Gamma-ray Lines and the Inclusive Photon Spectrum, Phys. Rev. D 86 (2012) 022002 [arXiv:1205.2739] [INSPIRE].

[31] J. Conrad, Searches for Particle Dark Matter with gamma-rays, AIP Conf. Proc. 1505 (2012) 166 [arXiv:1210.4392] [INSPIRE].

[32] Fermi-LAT collaboration, Search for Gamma-ray Spectral Lines with the Fermi Large Area Telescope and Dark Matter Implications, arXiv:1305.5597 [INSPIRE].

[33] H.E.S.S. collaboration, A. Abramowski et al., Search for photon line-like signatures from Dark Matter annihilations with H.E.S.S, Phys. Rev. Lett. 110 (2013) 041301 [arXiv: 1301.1173] [INSPIRE].

[34] LAT collaboration, M. Ackermann et al., Constraints on the Galactic Halo Dark Matter from Fermi-LAT Diffuse Measurements, Astrophys. J. 761 (2012) 91 [arXiv:1205.6474] [INSPIRE].

[35] D. Hooper, C. Kelso and F.S. Queiroz, Stringent and Robust Constraints on the Dark Matter Annihilation Cross Section From the Region of the Galactic Center, Astropart. Phys. 46 (2013) 55 [arXiv:1209.3015] [INSPIRE].

[36] Fermi-LAT collaboration, A. Abdo et al., Observations of Milky Way Dwarf Spheroidal galaxies with the Fermi-LAT detector and constraints on Dark Matter models, Astrophys. J. $\mathbf{7 1 2}$ (2010) 147 [arXiv: 1001.4531] [INSPIRE].

[37] Fermi LAT collaboration, A. Drlica-Wagner, Constraints on Dark Matter and Supersymmetry from LAT Observations of Dwarf Galaxies, arXiv:1210.5558 [INSPIRE].

[38] C. Weniger, A Tentative Gamma-Ray Line from Dark Matter Annihilation at the Fermi Large Area Telescope, JCAP 08 (2012) 007 [arXiv:1204.2797] [INSPIRE]. 
[39] T. Bringmann, X. Huang, A. Ibarra, S. Vogl and C. Weniger, Fermi LAT Search for Internal Bremsstrahlung Signatures from Dark Matter Annihilation, JCAP 07 (2012) 054 [arXiv: 1203.1312] [INSPIRE].

[40] E. Tempel, A. Hektor and M. Raidal, Fermi 130 GeV gamma-ray excess and dark matter annihilation in sub-haloes and in the Galactic centre, JCAP 09 (2012) 032 [Addendum ibid. 1211 (2012) A01] [arXiv: 1205.1045] [INSPIRE].

[41] G. Chalons and A. Semenov, Loop-induced photon spectral lines from neutralino annihilation in the NMSSM, JHEP 12 (2011) 055 [arXiv:1110.2064] [INSPIRE].

[42] G. Chalons, Gamma-ray lines constraints in the NMSSM, arXiv:1204.4591 [INSPIRE].

[43] S. Profumo and T. Linden, Gamma-ray Lines in the Fermi Data: is it a Bubble?, JCAP 07 (2012) 011 [arXiv: 1204.6047] [INSPIRE].

[44] A. Ibarra, S. Lopez Gehler and M. Pato, Dark matter constraints from box-shaped gamma-ray features, JCAP 07 (2012) 043 [arXiv: 1205.0007] [INSPIRE].

[45] XENON100 collaboration, E. Aprile et al., Dark Matter Results from 225 Live Days of XENON100 Data, Phys. Rev. Lett. 109 (2012) 181301 [arXiv:1207.5988] [INSPIRE].

[46] XENON100 collaboration, E. Aprile et al., Limits on spin-dependent WIMP-nucleon cross sections from 225 live days of XENON100 data, Phys. Rev. Lett. 111 (2013) 021301 [arXiv: 1301.6620] [INSPIRE].

[47] P. Beltrame on behalf of the XENON collaboration, Direct Dark Matter search with the XENON program, arXiv:1305.2719 [INSPIRE].

[48] A. Djouadi, A. Falkowski, Y. Mambrini and J. Quevillon, Direct Detection of Higgs-Portal Dark Matter at the LHC, Eur. Phys. J. C 73 (2013) 2455 [arXiv:1205.3169] [INSPIRE].

[49] CMS collaboration, Search for new physics in monojet events in pp collisions at $\sqrt{s}=8 \mathrm{TeV}$, CMS-PAS-EXO-12-048.

[50] J. Alwall, M. Herquet, F. Maltoni, O. Mattelaer and T. Stelzer, MadGraph 5: Going Beyond, JHEP 06 (2011) 128 [arXiv:1106.0522] [inSPIRE].

[51] T. Sjöstrand, S. Mrenna and P.Z. Skands, PYTHIA 6.4 Physics and Manual, JHEP 05 (2006) 026 [hep-ph/0603175] [INSPIRE].

[52] S. Ovyn, X. Rouby and V. Lemaitre, DELPHES, a framework for fast simulation of a generic collider experiment, arXiv:0903.2225 [INSPIRE].

[53] P.J. Fox, R. Harnik, J. Kopp and Y. Tsai, Missing Energy Signatures of Dark Matter at the LHC, Phys. Rev. D 85 (2012) 056011 [arXiv:1109.4398] [inSPIRE].

[54] CMS collaboration, Search for Dark Matter and Large Extra Dimensions in pp Collisions Yielding a Photon and Missing Transverse Energy, Phys. Rev. Lett. 108 (2012) 261803 [arXiv: 1204.0821] [INSPIRE].

[55] ATLAS collaboration, Search for dark matter candidates and large extra dimensions in events with a photon and missing transverse momentum in pp collision data at $\sqrt{s}=7 \mathrm{TeV}$ with the ATLAS detector, Phys. Rev. Lett. 110 (2013) 011802 [arXiv:1209.4625] [INSPIRE].

[56] W. Buchmüller and M. Garny, Decaying vs Annihilating Dark Matter in Light of a Tentative Gamma-Ray Line, JCAP 08 (2012) 035 [arXiv:1206.7056] [INSPIRE]. 